\title{
Hamiltonian Paths in Cartesian Powers of Directed Cycles
}

\author{
David Austin ${ }^{1}$, Heather Gavlas ${ }^{2}$, and Dave Witte ${ }^{3}$ \\ ${ }^{1}$ Department of Mathematics and Statistics, Grand Valley State University, Allendale, \\ MI 49401, USA. e-mail: austind@gvsu.edu \\ ${ }^{2}$ Department of Mathematics, Illinois State University, Normal, IL 61790-4520, USA. \\ e-mail: hgavlas@ilstu.edu \\ ${ }^{3}$ Department of Mathematics, Oklahoma State University, Stillwater, OK 74078, USA. \\ e-mail: dwitte@math.okstate.edu
}

\begin{abstract}
The vertex set of the $k^{\text {th }}$ cartesian power of a directed cycle of length $m$ can be naturally identified with the abelian group $\left(\mathbb{Z}_{m}\right)^{k}$. For any two elements $u=\left(u_{1}, \ldots, u_{k}\right)$ and $v=\left(v_{1}, \ldots, v_{k}\right)$ of $\left(\mathbb{Z}_{m}\right)^{k}$, it is easy to see that if there is a hamiltonian path from $u$ to $v$, then

$$
u_{1}+\cdots+u_{k} \equiv v_{1}+\cdots+v_{k}+1 \quad(\bmod m) \text {. }
$$
\end{abstract}

We prove the converse, unless $k=2$ and $m$ is odd.

\section{Introduction}

The cartesian product of any number of (undirected) cycles always contains a hamiltonian cycle. (See (2.2) for the definition of the cartesian product.) Work of Chen and Quimpo [2] implies the following stronger result, which provides a simple characterization of the pairs of vertices that can be joined by a hamiltonian path.

Theorem 1.1 (Chen-Quimpo [2]). Let $X$ be the cartesian product of $k$ cycles of lengths $m_{1}, m_{2}, \ldots, m_{k}$, with $k \geq 2$ and each $m_{i} \geq 3$.

(1) If some $m_{i}$ is odd, then $X$ is hamiltonian connected. That is, for any two vertices $u$ and $v$ of $X$, there is a hamiltonian path from $u$ to $v$.

(2) If each $m_{i}$ is even, then $X$ is hamiltonian laceable. That is, $X$ is bipartite and, for any two vertices $u$ and $v$ of $X$, either there is a hamiltonian path from $u$ to $v$, or there is a path of even length from $u$ to $v$.

It would be interesting to have a similar result in the directed case. The first step, which has been completed, is to determine which cartesian products of directed cycles have hamiltonian cycles. Rankin [5] implicitly gave a necessary 
and sufficient condition for the existence of a hamiltonian cycle in the cartesian product of two directed cycles; however, this result went unnoticed by graph theorists. Thirty years later, Trotter and Erdös [6] rediscovered the characterization. Curran and Witte [4] showed that there is a hamiltonian cycle in the cartesian product of three or more nontrivial directed cycles.

Theorem 1.2 (Rankin [5], Trotter-Erdös [6], and Curran-Witte [4]). Let $X$ be the cartesian product of $k$ directed cycles of lengths $m_{1}, m_{2}, \ldots, m_{k}$, with $k \geq 1$ and each $m_{i} \geq 2$. Then there is a hamiltonian cycle in $X$ if and only if either $k \neq 2$ or there exists a pair of relatively prime positive integers $s_{1}$ and $s_{2}$ with $s_{1} m_{1}+s_{2} m_{2}=m_{1} m_{2}$.

For the case $k=2$, Curran [4] strengthened Theorem 1.2 to obtain a description of the pairs of vertices that can be joined by a hamiltonian path in terms of the geometric configuration of the lattice points in the plane triangle with vertices $\left(m_{1}, 0\right),\left(0, m_{2}\right)$, and $(0,0)$. (Although the strengthened form of the case $k=2$ was published in the joint paper [4], the coauthor wishes to acknowledge that this important result was a solo effort of Curran, obtained before the coauthor joined the project.) The result is quite technical so, instead of stating it here, let us mention that when $k=2$, there always exist pairs of vertices that cannot be joined by a hamiltonian path. In fact, Curran showed, for each vertex $u$, that no more than half of the vertices of $X$ are the terminal vertex of some hamiltonian path starting at $u$.

In contrast, we conjecture that there is a hamiltonian path from $u$ to $v$ for any two distinct vertices $u$ and $v$ if $\operatorname{gcd}\left(m_{1}, m_{2}, \ldots, m_{k}\right)=1$ and $k \geq 3$. This is a special case of the following conjecture, which has no restriction on $m_{1}, m_{2}, \ldots, m_{k}$.

Notation 1.3. For vertices $u$ and $v$ in a (strongly connected) digraph $X$, let $d_{X}(u, v)$ denote the length of the shortest directed path from $u$ to $v$.

Conjecture 1.4. Let $X$ be the cartesian product of $k$ directed cycles of lengths $m_{1}, m_{2}, \ldots, m_{k}$, with $k \geq 3$ and each $m_{i} \geq 2$. For vertices $u$ and $v$ of $X$, there is a hamiltonian path from $u$ to $v$ if and only if

$$
d_{X}(u, v) \equiv-1\left(\bmod \operatorname{gcd}\left(m_{1}, m_{2}, \ldots, m_{k}\right)\right)
$$

For $X$ as in Conjecture 1.4, the lengths of any two directed paths from $u$ to $v$ are congruent modulo $\operatorname{gcd}\left(m_{1}, m_{2}, \ldots, m_{k}\right)$. This elementary observation implies that (1.5) is a necessary condition for the existence of a hamiltonian path from $u$ to $v$. Our conjecture is that it is also sufficient.

In this paper, we prove Conjecture 1.4 in the special case where all of the directed cycles have the same length.

Theorem 3.2'. Let $X$ be the $k^{\text {th }}$ cartesian power of a directed cycle of length $m$, with $k \geq 3$ and $m \geq 2$. For vertices $u$ and $v$ of $X$, there is a hamiltonian path from $u$ to $v$ if and only if

$$
d_{X}(u, v) \equiv-1(\bmod m)
$$




\section{Definitions and Preliminaries}

Let us begin this section with a few definitions. For background information about the graph theoretic terms used below, the reader is directed to [1]. For background information on hamiltonian cycles in Cayley digraphs, including the arc-forcing subgroup, see the surveys $[3,7]$.

Notation 2.1. The directed cycle on $m(m \geq 2)$ vertices is denoted by $\vec{C}_{m}$.

Definition 2.2. The cartesian product $G=G_{1} \times G_{2}$ of two digraphs $G_{1}$ and $G_{2}$ is the digraph whose vertex set is $V(G)=V\left(G_{1}\right) \times V\left(G_{2}\right)$ and has an arc from $\left(u_{1}, u_{2}\right)$ to $\left(v_{1}, v_{2}\right)$ if and only if either

$$
u_{1}=v_{1} \text { and there is an arc from } u_{2} \text { to } v_{2} \text { in } G_{2}
$$

or

$$
u_{2}=v_{2} \text { and there is an arc from } u_{1} \text { to } v_{1} \text { in } G_{1} \text {. }
$$

A convenient way of drawing $G_{1} \times G_{2}$ is to first place a copy of $G_{2}$ at each vertex of $G_{1}$ and then join corresponding vertices of $G_{2}$ in the copies of $G_{2}$ placed at adjacent vertices of $G_{1}$.

Notation 2.3. In the abelian group $\mathbb{Z}_{m_{1}} \times \mathbb{Z}_{m_{2}} \times \cdots \times \mathbb{Z}_{m_{k}}$, let

$$
\begin{aligned}
x_{1} & =(1,0,0, \ldots, 0), \\
x_{2} & =(0,1,0, \ldots, 0), \\
& \ldots \\
x_{k} & =(0,0, \ldots, 0,1) .
\end{aligned}
$$

The set $S=\left\{x_{1}, x_{2}, \ldots, x_{k}\right\}$ will denote the standard generating set of $\mathbb{Z}_{m_{1}} \times \mathbb{Z}_{m_{2}} \times \cdots \times \mathbb{Z}_{m_{k}}$.

Definition 2.4. Let $S$ generate a finite (abelian) group $\Gamma$. The Cayley digraph $\overrightarrow{\mathrm{Cay}}(\Gamma ; S)$ is the digraph whose vertex set is $V(G)=\Gamma$ and has an arc from $g$ to $g+s$ whenever $g \in \Gamma$ and $s \in S \backslash\{0\}$. (We delete 0 from $S$ to avoid having loops in the digraph.)

We may write $\overrightarrow{C a y}\left(\mathbb{Z}_{m_{1}} \times \mathbb{Z}_{m_{2}} \times \cdots \times \mathbb{Z}_{m_{k}}\right)$, omitting $S$ from the notation, when the standard generating set is to be used.

Notation 2.5. A path $P$ in a digraph can be specified by giving an ordered list $v_{0}, v_{1}, \ldots, v_{n}$ of the vertices encountered.

In a Cayley digraph $\overrightarrow{C a y}(\Gamma ; S)$, it is usually more convenient to specify the path $P: v_{0}, v_{1}, \ldots, v_{n}$ by giving the initial vertex $v_{0}$ and an ordered list $\left(a_{1}, a_{2}, \ldots, a_{n}\right)$ of elements of $S$ that label the arcs in $P$ as $v_{i}=v_{0}+a_{1}+\cdots+a_{i}$.

We will also use the notation $\left(a_{1}, a_{2}, \ldots, a_{n}\right)^{k}$ to indicate the concatenation of $k$ copies of the sequence $\left(a_{1}, a_{2}, \ldots, a_{n}\right)$. Thus, for example, $\left(a^{2}, b\right)^{3}=$ $(a, a, b, a, a, b, a, a, b)$. 
The following observation is well known (and easy to prove).

Proposition 2.6. If $\Gamma=\mathbb{Z}_{m_{1}} \times \mathbb{Z}_{m_{2}} \times \cdots \times \mathbb{Z}_{m_{k}}\left(m_{i} \geq 2\right)$ and $S$ is the standard generating set, then $\overrightarrow{C a y}(\Gamma ; S)$ is isomorphic to the cartesian product of $k$ directed cycles of lengths $m_{1}, m_{2}, \ldots, m_{k}$; that is,

$$
\overrightarrow{\operatorname{Cay}}\left(\mathbb{Z}_{m_{1}} \times \mathbb{Z}_{m_{2}} \times \cdots \times \mathbb{Z}_{m_{k}}\right) \cong \vec{C}_{m_{1}} \times \vec{C}_{m_{2}} \times \cdots \times \vec{C}_{m_{k}} .
$$

Thus, in order to understand cartesian products of directed cycles, it suffices to understand certain Cayley digraphs. This change of perspective provides an algebraic setting that makes some constructions more transparent. As Cayley digraphs are vertex-transitive, there is usually no harm in assuming that a hamiltonian path starts at the identity element. In $\overrightarrow{C a y}\left(\left(\mathbb{Z}_{m}\right)^{k}\right)$, there is a hamiltonian path from $v$ to $w$ if and only if there is a hamiltonian path from $0=(0,0, \ldots, 0)$ to $w-v$; thus throughout the rest of this paper, we will consider only that hamiltonian paths start at 0 .

The following subgroup of $\left(\mathbb{Z}_{m}\right)^{k}$, called the "arc-forcing subgroup," is a basic tool in the study of hamiltonian paths.

Definition 2.7. For the standard generating set $S$ of $\left(\mathbb{Z}_{m}\right)^{k}$, let $S^{-}=\{-s \mid s \in S\}$ and let

$$
\begin{aligned}
H & =\left\langle S+S^{-}\right\rangle \\
& =\left\{\left(v_{1}, \ldots, v_{k}\right) \in\left(\mathbb{Z}_{m}\right)^{k} \mid v_{1}+\cdots+v_{k} \equiv 0(\bmod m)\right\} .
\end{aligned}
$$

Then $H$ is called the arc-forcing subgroup.

Remark 2.8. Let us recall some basic facts.

- For any $s_{1}, s_{2}, x \in S$, we have

$$
s_{1}-s_{2}=\left(s_{1}-x\right)-\left(s_{2}-x\right) \in\langle S-x\rangle,
$$

where $S-x=\{s-x \mid s \in S\}$. Therefore $S-x$ is a generating set for $H$.

- For $v=\left(v_{1}, v_{2}, \ldots, v_{k}\right) \in\left(\mathbb{Z}_{m}\right)^{k}$, we have $v \in H-x_{1}$ if and only if $v_{1}+v_{2}+$ $\ldots+v_{k} \equiv-1 m$.

- If there is a hamiltonian path from 0 to $v$ in $\overrightarrow{C a y}\left(\left(\mathbb{Z}_{m}\right)^{k}\right)$, then $v \in H-x_{1}$.

- $\overrightarrow{\operatorname{Cay}}\left(H ; S-x_{1}\right) \cong \overrightarrow{\mathrm{Cay}}\left(\left(\mathbb{Z}_{m}\right)^{k-1}\right)$.

\section{Hamiltonian Paths}

Notation 3.1. Throughout this section:

- $m$ and $k$ are positive integers with $m, k \geq 2$, 
- $S$ is the standard generating set of $\left(\mathbb{Z}_{m}\right)^{k}$, say $S=\left\{x_{1}, x_{2}, \ldots, x_{k}\right\}$, and

- $H=\left\langle S+S^{-}\right\rangle$is the arc-forcing subgroup.

In this section, we will prove our main result.

Theorem 3.2. If $k \geq 3$, then for every $v \in H-x_{1}$, there is a hamiltonian path from 0 to $v$ in $\overrightarrow{\mathrm{Cay}}\left(\left(\mathbb{Z}_{m}\right)^{k}\right)$.

Since $\overrightarrow{\mathrm{Cay}}\left(H ; S-x_{1}\right) \cong \overrightarrow{\mathrm{Cay}}\left(\left(\mathbb{Z}_{m}\right)^{k-1}\right)$, it is easy to see that $\overrightarrow{\mathrm{Cay}}\left(H ; S-x_{1}\right)$ contains a hamiltonian cycle $C$. If $v$ is any element of $H$ that is an even distance from 0 along $C$, then the following proposition shows that there is a hamiltonian path from 0 to $v-x_{1}$ in $\overrightarrow{C a y}\left(\left(\mathbb{Z}_{m}\right)^{k}\right)$. This observation is the main tool in our proof of Theorem 3.2.

Proposition 3.3. Let $v \in H$.If there is a hamiltonian cycle $C$ in $\overrightarrow{C a y}\left(H ; S-x_{1}\right)$ suchthat $d_{C}(0, v)$ is even, then $\overrightarrow{C a y}\left(\left(\mathbb{Z}_{m}\right)^{k}\right)$ contains a hamiltonian path from 0 to $v-x_{1}$.

Proof. Let $C: c_{0}, c_{1}, \ldots, c_{m^{k-1}}$ be a hamiltonian cycle in $\overrightarrow{\mathrm{Cay}}\left(H ; S-x_{1}\right)$ with $c_{0}=c_{m^{k-1}}=0$, and define

$$
\phi: \mathbb{Z}_{m} \times \mathbb{Z}_{m^{k-1}} \rightarrow\left(\mathbb{Z}_{m}\right)^{k} \text { by } \phi(i, j)=i x_{1}+c_{j} .
$$

For convenience, let $a=(1,0)$ and $b=(1,1)$ in $\mathbb{Z}_{m} \times \mathbb{Z}_{m^{k-1}}$. Then, for any $v=(i, j) \in \mathbb{Z}_{m} \times \mathbb{Z}_{m^{k-1}}$, we have

$$
\phi(v+a)-\phi(v)=x_{1} \in S
$$

and

$$
\phi(v+b)-\phi(v)=x_{1}+\left(c_{j+1}-c_{j}\right) \in S,
$$

as $c_{j+1}-c_{j} \in S-x_{1}$. So $\overrightarrow{C a y}\left(\left(\mathbb{Z}_{m}\right)^{k}\right)$ contains arcs from $\phi(v)$ to $\phi(v+a)$ and $\phi(v+b)$. Therefore, $\phi$ embeds $\overrightarrow{C a y}\left(\mathbb{Z}_{m} \times \mathbb{Z}_{m^{k-1}} ;\{a, b\}\right)$ as a spanning subdigraph of $\left.\overrightarrow{\mathrm{Cay}}\left(\left(\mathbb{Z}_{m}\right)^{k}\right)\right)$. Now, for $0 \leq n<m^{k-1} / 2$, the path

$$
\left(\left(a^{m-2}, b^{2}\right)^{n},\left(a^{m-2}, b, a\right)^{m^{k-1}-2 n-1},\left(a^{m-2}, b^{2}\right)^{n}, a^{m-2}, b\right)
$$

is a hamiltonian path $P$ from 0 to $(-1,2 n)$ in $\overrightarrow{\mathrm{Cay}}\left(\mathbb{Z}_{m} \times \mathbb{Z}_{m^{k-1}} ;\{a, b\}\right)$. So $\phi(P)$ is a hamiltonian path from $\phi(0)=0$ to $\phi(-1,2 n)=c_{2 n}-x_{1}$ in $\overrightarrow{\mathrm{Cay}}\left(\left(\mathbb{Z}_{m}\right)^{k}\right)$.

The following corollary establishes Theorem 3.2. in the case where $m$ is even.

Corollary 3.4. If $m$ is even, then for every $v \in H-x_{1}$, there is a hamiltonian path from 0 to $v$ in $\overrightarrow{C a y}\left(\left(\mathbb{Z}_{m}\right)^{k}\right)$. 
Proof. Let $v=\left(v_{1}, v_{2}, \ldots, v_{k}\right)$. Since $v \in H-x_{1}$, we have that $v_{1}+v_{2}+\cdots+$ $v_{k} \equiv-1(m)$. Since $m$ is even, it must be the case that $v_{i}$ is odd for some $i$; without loss of generality, say $v_{1}$ is odd. We may assume this without loss of generality as $S-x_{i}$ is a generating set for $H$ and $v \in H-x_{i}$ for each $i(1 \leq i \leq k)$. Let $C$ be a hamiltonian cycle in $\overrightarrow{\mathrm{Cay}}\left(H ; S-x_{1}\right)$.

For $\epsilon \in\{0,1\}$, let

$$
H_{\epsilon}=\left\{h \in H \mid h_{1} \equiv \epsilon(\bmod 2)\right\} .
$$

Then $H_{0}$ and $H_{1}$ form a bipartition of $\overrightarrow{\mathrm{Cay}}\left(H ; S-x_{1}\right)$. Therefore, since $0 \in H_{0}$, we know that $d_{C}(0, h)$ is even for every $h \in H_{0}$. Also, since $v+x_{1} \in H$ and the first coordinate of $v+x_{1}$ is even, we know that $v+x_{1} \in H_{0}$. Hence $d_{C}\left(0, v+x_{1}\right)$ is even, and thus by Proposition 3.3 , there is a hamiltonian path in $\overrightarrow{\mathrm{Cay}}\left(\left(\mathbb{Z}_{m}\right)^{k}\right)$ from 0 to $\left(v+x_{1}\right)-x_{1}=v$.

Observe that Corollary 3.4 applies for all $k$, whenever $m$ is even. In contrast, the following remark shows that if $k=2$ and $m$ is odd, then only $(m+1) / 2$ of the $m$ elements of $H-x_{1}$ are the terminal endpoint of a hamiltonian path that starts at 0 .

Remark 3.5. Assume $k=2$ and $m$ is odd. Let $v \in H-x_{1}$, and write $v=\left(v_{1}, v_{2}\right)$ with $0 \leq v_{1}, v_{2}<m$. Then $v_{1}+v_{2}=m-1$, which is even. Hence $v_{1}$ and $v_{2}$ have the same parity. The work of Curran [4] shows that there is a hamiltonian path from 0 to $v$ in $\overrightarrow{\mathrm{Cay}}\left(\left(\mathbb{Z}_{m}\right)^{2}\right)$ if and only if $v_{1}$ is even. Thus, if $m$ is odd, then the assumption that $k \geq 3$ is necessary in Theorem 3.2.

We now wish to apply Proposition 3.3 in the case that $m$ is odd. We begin by showing that we can find the appropriate hamiltonian cycle.

Lemma 3.6. Assume that $m$ is odd and that $n$ is a multiple of $m$. Let $v \in \mathbb{Z}_{m} \times \mathbb{Z}_{n}$ where $v=(i, j)$ with $0 \leq i<m$ and $0 \leq j<n$, and let $r$ be the remainder of $i+$ jupon division by $m$. If either

(1) $j+r$ is even, or

(2) both $j$ and $r$ are nonzero, or

(3) $j$ is even and nonzero,

then there is a hamiltonian cycle $C$ in $\overrightarrow{\mathrm{Cay}}\left(\mathbb{Z}_{m} \times \mathbb{Z}_{n}\right)$ such that $d_{C}(0, v)$ is even.

Proof. As usual, let $x_{1}=(1,0)$ and $x_{2}=(0,1)$.

(1) If $j+r$ is even, let $C$ be the hamiltonian cycle $\left(x_{1}^{m-1}, x_{2}\right)^{n}$. Then, since

$$
v=i x_{1}+j x_{2}=j\left((m-1) x_{1}+x_{2}\right)+r x_{1},
$$

we have that

$$
d_{C}(0, v)=j m+r \equiv j+r \equiv 0(\bmod 2) .
$$

Hence $d_{C}(0, v)$ is even. 
(2) If $j+r$ is odd, and both $j$ and $r$ are nonzero, let $C$ be the hamiltonian cycle $\left(x_{2}, x_{1}^{m-1}\right)^{n}$. Then, since

$$
v=i x_{1}+j x_{2}=(j-1)\left(x_{2}+(m-1) x_{1}\right)+x_{2}+(r-1) x_{1},
$$

we have that

$$
d_{C}(0, v)=(j-1) m+1+(r-1) \equiv(j-1)+r \equiv 0(\bmod 2) .
$$

Hence $d_{C}(0, v)$ is even.

(3) Suppose $j$ is even and nonzero. If $j+r$ is even, then (1) applies. If not, then $r$ must be odd, so $r \neq 0$. Therefore (2) applies.

Proposition 3.7. If $m$ is odd and $n \geq 2$, then, for each $v \in\left(\mathbb{Z}_{m}\right)^{n}$, there is a hamiltonian cycle $C$ in $\overrightarrow{C a y}\left(\left(\mathbb{Z}_{m}\right)^{n}\right)$ such that $d_{C}(0, v)$ is even.

Proof. The proof is by induction on $n$.

Base Case. Assume that $n=2$. Write $v=i x_{1}+j x_{2}$ for some $i$ and $j$ with $0 \leq i, j \leq m-1$. Let $r$ be the remainder of $i+j$ upon division by $m$. We may assume that $i+r$ and $j+r$ are both odd, for otherwise the desired conclusion follows from Lemma 3.6(1) (perhaps after interchanging $i$ and $j$ ). This implies that $i$ and $j$ have the same parity, so $i+j$ is even. The desired conclusion is obvious if $v=0$, so we may assume that not both of $i$ and $j$ are 0 ; by symmetry, we may assume $j \neq 0$. Now $i+j \neq 0$ and, because $i+j$ is even, we know $i+j \neq m$; therefore $r \neq 0$. Thus, the desired conclusion follows from Lemma 3.6(2).

Induction Step. Let $n \geq 3$. Assume for every vertex $w$ of $\overrightarrow{C a y}\left(\left(\mathbb{Z}_{m}\right)^{n-1}\right)$, there is a hamiltonian cycle $C$ such that $d_{C}(0, w)$ is even. Write $v=\left(v_{1}, \ldots, v_{n}\right)$. We may assume $v \neq 0$, for otherwise the desired conclusion is obvious. By symmetry, we may assume that $v_{n} \neq 0$. Let $w=\left(v_{2}, \ldots, v_{n}\right)$. By induction, there is a hamiltonian cycle $C_{0}: c_{0}, c_{1}, \ldots, c_{m^{n-1}}$ in $\overline{\mathrm{Cay}}\left(\left(\mathbb{Z}_{m}\right)^{n-1}\right)$ with $c_{0}=c_{m^{n-1}}=0$, such that $d_{C_{0}}(0, w)$ is even. Let $c_{\ell}=w$. Define

$$
\phi: \mathbb{Z}_{m} \times \mathbb{Z}_{m^{n-1}} \rightarrow\left(\mathbb{Z}_{m}\right)^{n} \text { by } \phi(i, j)=i x_{1}+\left(0, c_{j}\right)
$$

Then, for any $u=(i, j) \in \mathbb{Z}_{m} \times \mathbb{Z}_{m^{n-1}}$, we have

$$
\phi(u+(1,0))-\phi(u)=x_{1} \in S
$$

and

$$
\phi(u+(0,1))-\phi(u)=\left(0, c_{j+1}\right)-\left(0, c_{j}\right) \in S,
$$

$\underline{\text { as }} c_{j+1}-c_{j}$ is an element of the standard generating set for $\left(\mathbb{Z}_{m}\right)^{n-1}$. So $\overrightarrow{\mathrm{Cay}}\left(\left(\mathbb{Z}_{m}\right)^{n}\right)$ contains arcs from $\phi(u)$ to $\phi(u+(1,0))$ and $\phi(u+(0,1))$. Therefore, $\phi$ embeds $\overrightarrow{\mathrm{Cay}}\left(\mathbb{Z}_{m} \times \mathbb{Z}_{m^{n-1}}\right)$ as a spanning subdigraph of $\overrightarrow{\mathrm{Cay}}\left(\left(\mathbb{Z}_{m}\right)^{n}\right)$, with 
$\phi(0)=0$ and $\phi\left(v_{1}, \ell\right)=v$. Because $\ell$ is even and nonzero, Lemma 3.6(3) implies that there is a hamiltonian cycle $C$ in $\overrightarrow{\mathrm{Cay}}\left(\mathbb{Z}_{m} \times \mathbb{Z}_{m^{n-1}}\right)$, such that $d_{C}\left(0,\left(v_{1}, \ell\right)\right)$ is even. Then $\phi(C)$ is a hamiltonian cycle in $\overrightarrow{\mathrm{Cay}}\left(\left(\mathbb{Z}_{m}\right)^{n}\right)$ such that $d_{\phi(C)}(0, v)$ is even.

Combining the results of Propositions 3.3 and 3.7 together with Corollary 3.4, we now give the proof of our main result.

Proof of Theorem 3.2. If $m$ is even, then the desired conclusion follows by Corollary 3.4. Thus, we may assume that $m$ is odd. Since $\overrightarrow{C a y}\left(H ; S-x_{1}\right)$ is isomorphic to $\overrightarrow{C a y}\left(\left(\mathbb{Z}_{m}\right)^{k-1}\right)$, Proposition 3.7 implies that there is a hamiltonian cycle $C$ in $\overrightarrow{C a y}\left(H ; S-x_{1}\right)$ such that $d_{C}\left(0, v+x_{1}\right)$ is even. The desired conclusion now follows from Proposition 3.

Unfortunately, our methods do not seem to be sufficient to prove the general case of Conjecture 1.4. For example, Lemma 3.6 does not seem to be useful unless $m_{i}$ is a divisor of $\left(m_{1} m_{2} \cdots m_{k}\right) / m_{i}$, for some $i$.

Acknowledgments. D.W. was partially supported by a grant from the National Science Foundation (DMS-9801136).

\section{References}

1. Chartrand, G., Lesniak, L.: Graphs \& Digraphs, Third Edn, London: Chapman-Hall, 1996

2. Chen, C.C., Quimpo, N.F.: On strongly hamiltonian abelian group graphs. In: K.L. McAvaney: Combinatorial Mathematics VIII, Lect. Notes Math. Vol. 884, pp. 23-34. Berlin: Springer-Verlag, 1981

3. Curran, S.J., Gallian, J.A.: Hamiltonian cycles and paths in Cayley graphs and digraphs-a survey. Discrete Math. 156, 1-18 (1996)

4. Curran, S.J., Witte, D.: Hamilton paths in cartesian products of directed cycles. Ann. Discrete Math. 27, 35-74 (1985)

5. Rankin, R.A.: A campanological problem in group theory. Proc. Camb. Phil. Soc. 44, 17-25 (1948)

6. Trotter, W., Erdös, P.: When the cartesian product of directed cycles is hamiltonian. J. Graph Theory 2, 137-142 (1978)

7. Witte, D., Gallian, J. A.: A survey: hamiltonian cycles in cayley graphs. Discrete Math. 51, 293-304 (1984)

Received: October 25, 2001

Final version received: June 11,2002 